\title{
Motoneuron Survival Is Enhanced in the Absence of Neuromuscular Junction Formation in Embryos
}

\author{
J. Terrado, ${ }^{1}$ R. W. Burgess, ${ }^{2}$ T. DeChiara, ${ }^{3}$ G. Yancopoulos, ${ }^{3}$ J. R. Sanes, ${ }^{2}$ and A. C. Kato ${ }^{1}$ \\ ${ }^{1}$ Division of Clinical Neuromuscular Research and Department of Anesthesiology, Pharmacology and Surgical Intensive \\ Care, Faculty of Medicine, Centre Médical Universitaire, 1211 Geneva 4, Switzerland, 2Department of Anatomy and \\ Neurobiology, Washington University Medical School, St. Louis, Missouri 63110, and ${ }^{3}$ Regeneron Pharmaceuticals, \\ Tarrytown, New York 10591
}

\begin{abstract}
Approximately half of the motoneurons produced during development die before birth or shortly after birth. Although it is believed that survival depends on a restricted supply of a trophic sustenance produced by the synaptic target tissue (i.e., muscle), it is unclear whether synapse formation per se is involved in motoneuron survival. To address this issue, we counted cranial motoneurons in a set of mutant mice in which formation of neuromuscular junctions is dramatically impaired (i.e., null mutants for agrin, nerve-derived agrin, rapsyn, and MuSK).

We demonstrate that in the absence of synaptogenesis, there is an $18-34 \%$ increase in motoneuron survival in the facial, trochlear, trigeminal motor, and hypoglossal nuclei; the highest survival occurred in the MuSK-deficient animals in which synapse formation is most severely compromised. There was no
\end{abstract}

change in the size of the mutant motoneurons as compared with control animals, and the morphology of the mutant motoneurons appeared normal. We postulate that the increased axonal branching observed in these mutants leads to a facilitated "access" of the motoneurons to muscle-derived trophic factors at sites other than synapses or that inactivity increases the production of such factors. Finally, we examined motoneurons in double mutants of CNTFR $\alpha^{-1-}$ (in which there is a partial loss of motoneurons) and $\mathrm{MuSK}^{-1-}$ (in which there is an increased survival of motoneurons). The motoneuron numbers in the double mutants parallel those of the single MuSKdeficient mice, indicating that synapse disruption can even overcome the deleterious effect of CNTFR $\alpha$ ablation.

Key words: motoneuron survival; neuromuscular junction formation; null mutant mice; rapsyn; MuSK; agrin
More neurons are produced in embryos than are present in adults, the difference reflecting naturally occurring cell death during a defined developmental period (Oppenheim, 1991; Henderson, 1996). An important influence on whether a neuron lives or dies appears to be the extent to which it receives adequate trophic sustenance from tissues to which its axon projects. For example, supernormal numbers of sensory and motor neurons died after ablation of limb buds to which these neurons would normally project. Conversely, transplantation of a supernumerary limb rescued a substantial fraction (although not all) of the neurons that would otherwise have died (for review, see Oppenheim, 1991). Subsequent studies identified a variety of trophic factors that are synthesized by target tissues and capable of promoting survival of cultured neurons (for review, see Oppenheim, 1991; Henderson, 1996; Lewin and Barde, 1996).

A critical question that remains unanswered is how the amount of trophic support a neuron receives is regulated. For motoneurons, trophic support is provided by muscles, which are synaptic

Received Oct. 9, 2000; revised Feb. 5, 2001; accepted Feb. 8, 2001.

This work was supported by the Association Française contre les Myopathies (France) and the Swiss National Science Foundation (A.C.K. and J.T.), and by the National Institutes of Health (J.R.S. and R.W.B.). A Spanish version of this work has been awarded the Coris Gruart Prize to J.T. We thank B. King, D. Monnier, D. Perrelet, and R. Vejsada for their assistance throughout this work.

Correspondence should be addressed to Ann Kato, Division of Clinical Neuromuscular Research and Department of Anesthesiology, Pharmacology and Surgical Intensive Care, Centre Médical Universitaire, 1 rue Michel Servet, 1211 Geneva 4, Switzerland. E-mail: Ann.Kato@medecine.unige.ch.

Dr. Terrado's present address: Departamento de Medicina y Cirugía Animal. Facultad Ciencias Experimentales y de la Salud. Universidad Cardenal HerreraCEU. 46113, Moncada-Valencia, Spain.

Copyright (C) 2001 Society for Neuroscience $0270-6474 / 01 / 213144-07 \$ 15.00 / 0$ targets, so it is reasonable to imagine that synapses are involved. Indeed, chronic pharmacological blockade of neuromuscular activity results in the rescue of motoneurons that would otherwise die, whereas stimulation of motor axons at higher than normal levels increases cell death (for review, see Oppenheim, 1991). These results imply that synaptic activity affects motoneuronal survival. In contrast, it is unclear whether synapse formation per se is essential for survival of motoneurons. Here, we used several strains of mutant mice to address this issue.

The mutants we used bear targeted mutations of the agrin, rapsyn, and MuSK genes. Agrin is a critical nerve-derived organizer of postsynaptic differentiation at the skeletal neuromuscular junction, MuSK is a component of the agrin receptor, and rapsyn is a cytoplasmic protein required for clustering of acetylcholine receptors (AChRs) and other signaling components in the postsynaptic membrane (Gautam et al., 1995, 1996, 1999; DeChiara et al., 1996). Accordingly, mutants lacking any of these three proteins display similar (but not identical) devastating defects in postsynaptic differentiation and die at birth because they cannot breathe. In addition, presynaptic differentiation is disrupted in all three mutants (Gautam et al., 1995, 1996, 1999; DeChiara et al., 1996; Burgess et al., 1999). The presynaptic defects appear likely to be indirect consequences of the postsynaptic defects, in that MuSK and rapsyn are expressed in muscle fibers but not neurons. Thus, the lack of postsynaptic differentiation presumably interferes with the generation or presentation of a retrograde signal that leads to presynaptic differentiation (for review, see Sanes et al., 1998; Sanes and Lichtman, 1999).

The fact that motor axons are present in these mutants indi- 
Table 1. Number of motoneurons in agrin null mutant mice (agrin $^{-/-}$) and their wild-type and heterozygous (control) littermates

\begin{tabular}{lccc} 
& Control & Agrin $^{-/-}$ & $\%$ increase \\
\hline Trochlear & $215 \pm 12(11)$ & $271 \pm 13(7)^{* *}$ & 26 \\
Trigeminal motor & $1073 \pm 37(9)$ & $1339 \pm 58(6)^{* *}$ & 25 \\
Facial & $4490 \pm 200(5)$ & $4734 \pm 89(5)$ & 5 \\
Hypoglossal & $2024 \pm 79(2)$ & $2412 \pm 98(3)$ & 19 \\
\hline
\end{tabular}

Control and agrin ${ }^{-1-}$ values are the average \pm SEM. The values in parentheses indicate the number of animals examined $(* * p<0.01)$. Percentages $(\%)$ refer to the increase in the number of motoneurons with respect to the controls.

cated that at least some motoneurons survived. It was not clear from previous work, however, whether the number of surviving motoneurons was normal, greatly reduced, or even larger than normal. To distinguish these alternatives, we analyzed the number of motoneurons in several cranial motor nuclei (trochlear, trigeminal, facial, and hypoglossal) of mutant mice lacking all forms of agrin, only nerve-derived (active) agrin, MuSK, or rapsyn, and their littermate controls. We report here that numbers of motoneurons were greater than normal in all four nuclei of all four mutant strains. Thus, motoneurons can survive without making neuromuscular junctions and therefore must have nonsynaptic means of acquiring trophic sustenance from their targets.

\section{MATERIALS AND METHODS}

Animals. Mice bearing targeted mutations in the genes for MuSK (DeChiara et al., 1996), rapsyn (Gautam et al., 1995), and CNTFR $\alpha$ (DeChiara et al., 1995) have been described previously. Two different agrin-deficient mutants were examined; one is a null mutant (R. W. Burgess and J. R. Sanes, unpublished observations) that has a phenotype which is identical to that reported for a severe hypomorph (Gautam et al., 1996), and the other has a deletion of the nerve-derived $\mathrm{z}$ form of agrin (Burgess et al., 1999). All lines (C57/B6) were maintained as heterozygotes and crossed to obtain homozygotes.

The genes encoding MuSK and CNTFR $\alpha$ both reside on mouse chromosome 4, and these are located 26.3 and $19.9 \mathrm{~cm}$, respectively, from the centromere (Valenzuela et al., 1995). To generate compound heterozygous mutant mice by spontaneous intrachromosomal recombination, we crossed MuSK ${ }^{+/-}$and CNTFR $\alpha^{+/-}$mice and observed that $\sim 20 \%$ of the progeny were heterozygous for both genes. These compound heterozygotes were then crossed to generate $\mathrm{MuSK}^{-1-}$ and CNTFR $\alpha^{-1-}$ double mutants.

Histology. Brains of newborn mice were fixed in Bouin's solution, dehydrated in a graded series of alcohol solutions, and embedded in paraffin. The brains were serially sectioned $(8 \mu \mathrm{m})$ and stained with cresyl violet. Motoneurons of the different motor nuclei with a clearly identifiable nucleus and nucleolus were counted in every fifth section (Terrado et al., 2000a,b). We compared motoneuron numbers in mice with synapse disruption (i.e., mice homozygous for the different mutations) with control mice with normal synapse formation using an unpaired Student's $t$ test. Because we did not observe phenotypic differences or variations in the number of motoneurons between wild-type and heterozygous animals, we pooled the values of these mice when indicated (Tables 1 and 2). Results are expressed as the mean \pm SEM.

Cross-sectional area of motoneurons was measured in 8 - $\mu$ m-thick sections stained with cresyl violet using a PC-assisted image analysis system (Zeiss KS 400 Imaging System). Only motoneuron profiles containing a distinct nucleus with nucleolus were included.

Embryonic day (E) 18 embryos were fixed in 4\% paraformaldehyde in PBS for $1 \mathrm{hr}$ at room temperature. Whole masseter muscles or cryostat sections of tongue muscle were then stained as described by Burgess et al. (1999). Briefly, axons were stained with a mixture of mouse monoclonal anti-neurofilament (Sternberger Monoclonals Inc., Exeter, UK) and antiSV2 antibodies (Developmental Studies Hybridoma Bank, Iowa City, IA), and AChRs were stained with rhodamine- $\alpha$-bungarotoxin (Molecular Probes, Eugene, OR). The monoclonal antibodies were visualized with fluorescein-conjugated goat anti-mouse IgG1 (Boehringer Mannheim, Indianapolis, IN). Muscles were viewed and photographed with rhodamine- and fluorescein-selective filters.
Table 2. Number of motoneurons in z-specific agrin mutant mice $\left(\operatorname{agrin}^{\Delta z / \Delta z}\right)$ and their wild-type and heterozygous (control) littermates

\begin{tabular}{lccl} 
& Control & \multicolumn{1}{c}{$\operatorname{Agrin}^{\Delta z / \Delta z}$} & $\%$ increase \\
\hline Trochlear & $171 \pm 11(7)$ & $259 \pm 13(5)^{* * *}$ & 51 \\
Trigeminal motor & $1180 \pm 50(6)$ & $1380 \pm 64(6)^{*}$ & 17 \\
Facial & $4530 \pm 178(6)$ & $4964 \pm 169(5)$ & 10 \\
Hypoglossal & $1964 \pm 164(2)^{a}$ & $2518(1)$ & 28 \\
\hline
\end{tabular}

$\overline{\text { Control and agrin }}{ }^{\Delta z / \Delta z}$ values are the average \pm SEM. The values in parentheses indicate the number of animals examined $\left({ }^{*} p<0.05,{ }^{*} * p<0.001\right)$. Percentages $(\%)$ refer to the increase in the number of motoneurons with respect to the controls. ${ }^{a}$ The control values were 1800 and 2128 hypoglossal motoneurons.

\section{RESULTS}

\section{Neuromuscular defects in muscles innervated by cranial motoneurons}

Cranial motor nuclei are advantageous for studies of factors that affect neuronal number because their limits, unlike those of spinal motor columns, are readily defined. However, previous analyses of agrin-, rapsyn-, and MuSK-deficient mutant mice have documented only neuromuscular defects in muscles innervated by spinal motoneurons (limb and axial muscles) (Gautam et al., 1995, 1996, 1999; DeChiara et al., 1996). We therefore began the present study by assessing the mutant phenotypes in two muscles supplied by cranial nuclei: the tongue, which is innervated by motoneurons in the hypoglossal nucleus, and the masseter, which is innervated by motoneurons in the trigeminal nucleus. Whole muscles and sections were doubly stained with antibodies to neural antigens to label motor axons (visualized with fluorescein-conjugated second antibodies) and with rhodamine- $\alpha$-bungarotoxin to label AChRs in the postsynaptic membrane.

Examples from a rapsyn ${ }^{-1-}$ mutant and a littermate control are shown in Figure 1; similar results have been obtained in $\mathrm{MuSK}^{-1-}$ and agrin ${ }^{-1-}$ mutants (data not shown). In control masseter muscle, axons form a central nerve trunk; individual axons branch to form rudimentary arbors on myotubes, with each presynaptic arbor (Fig. $1 A, B$ ) being precisely apposed to an AChR-rich patch of postsynaptic membrane (Fig. $1 A^{\prime}$ ). In homozygous mutants, in sharp contrast, axons entered the muscle normally, but they grew excessively and failed to form arbors (Fig. $1 D, E)$; moreover, no AChR clusters were ever detected on the mutant myotubes (Fig. 1 $\left.D^{\prime}\right)$. Similar results were obtained in tongue muscle in which the large number of synaptic sites in the control made the postsynaptic defect in the mutant especially dramatic (Fig. $1 C, F$ ). In all respects, presynaptic and postsynaptic defects observed in masseter and tongue were similar to those previously described for limb and axial muscles (Gautam et al., 1995, 1996; DeChiara et al., 1996; Burgess et al., 1999).

\section{Motoneuron survival in agrin-deficient mice}

We initially counted motoneurons in mice lacking all agrin isoforms (termed agrin ${ }^{-1-}$ ). Agrin-deficient mutant mice are unable to move, and they die in utero or very soon after birth (Gautam et al., 1996; Burgess et al., 1999). No phenotypic abnormality was seen in the heterozygous littermates (Gautam et al., 1996). The number of motoneurons was greater in all four cranial nuclei analyzed from these mutants than in the corresponding nuclei of littermate controls agrin $^{+/-}$and $^{\text {agrin }}{ }^{+/+}$) (Table 1). A statistically significant increase was observed in the number of motoneurons in the trochlear $(26 \%)$ and trigeminal $(25 \%)$ motor nuclei. Although the difference in the facial and hypoglossal 

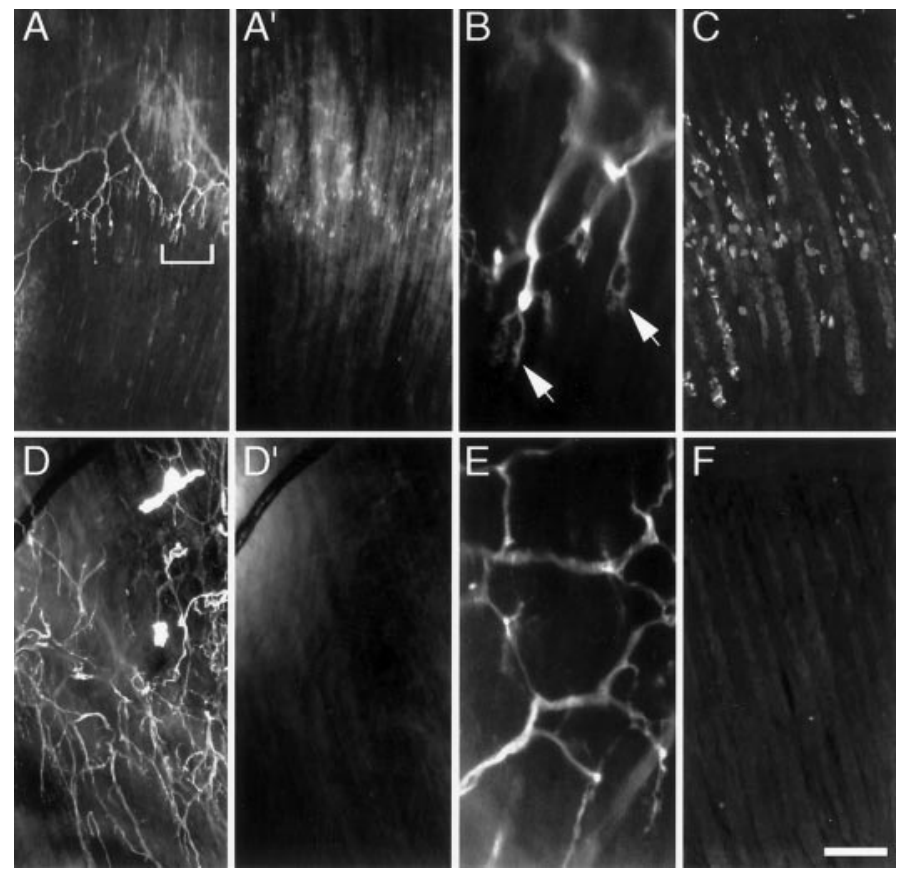

Figure 1. Defective neuromuscular development in cranial muscles of rapsyn mutants. Whole masseter muscles $\left(A, A^{\prime}, B, D, D^{\prime}, E\right)$ and sections of tongue muscle $(C, F)$ from embryonic day (E) 18 control $(A-C)$ and rapsyn $^{-1-}$ mutant mice $(D-F)$ were stained with fluorescein-conjugated anti-neurofilament and anti-SV2 to visualize nerves $(A, B, D, E)$, and with rhodamine- $\alpha$-bungarotoxin to visualize AChRs $\left(A^{\prime}, C, D^{\prime}, F\right)$. In controls, axons form rudimentary terminal arbors on AChR-rich patches of myotube membrane. In mutants, motor axons grow excessively and fail to form terminal arbors, and no AChR-rich clusters are present on myotubes. Region marked by bracket in $A$ is shown at higher magnification in $B$; arrows in $B$ indicate rudimentary terminal arbors. Scale bar (shown in $F)$ : $A, A^{\prime}, C, D, D^{\prime}, F, 100 \mu \mathrm{m} ; B, E, 25 \mu \mathrm{m}$.

nuclei was not statistically significant, there was a trend toward an increased motoneuron survival.

Motoneuron-derived agrin ( $\mathrm{z}^{+}$-agrin) is $100-$ to 1000 -fold more potent in aggregating AChRs than muscle- or glial-derived agrin and is essential for synapse formation (Burgess et al., 1999). Nevertheless the fact that agrin is also expressed by muscle and glial cells raises the possibility that the increase in motoneuron number observed in agrin ${ }^{-/-}$mice was caused by the deletion of non-neuronal agrin. To test this hypothesis, we analyzed a second strain of agrin-deficient mice (termed $\operatorname{agrin}^{\Delta z / \Delta z}$ ) in which only the neuronal isoform of agrin, $\mathrm{z}^{+}$-agrin, had been deleted.These mice exhibited the same phenotype as those in which agrin was ubiquitously eliminated, and there were no phenotypic differences between wild types and heterozygotes (Burgess et al., 1999).

In $\operatorname{agrin}^{\Delta z / \Delta z}$ mice, we also found an enhanced survival of cranial motoneurons in the four motor nuclei that were analyzed (Table 2). The biggest increase was observed in the trochlear nucleus, which contained $51 \%$ more motoneurons in mutants than in control littermates, followed by the trigeminal motor nucleus, which showed a $17 \%$ increase. In the facial nucleus, there was a $10 \%$ augmentation in motoneuron number, but this value did not differ significantly from the controls. In addition, we counted the hypoglossal nucleus in one animal, and there were also more motoneurons $(28 \%)$ than in the control. These results indicate that the absence of neuronal agrin is sufficient to reduce cell death of cranial motoneurons. Because the main role of
Table 3. Number of motoneurons in MuSK mutant mice $\left(\mathrm{MuSK}^{-1-}\right)$ and their wild-type littermates

\begin{tabular}{lccl} 
& $\mathrm{MuSK}^{+/+}$ & $\mathrm{MuSK}^{-/-}$ & $\%$ increase \\
\hline Trochlear & $218 \pm 7(6)$ & $297 \pm 17(8)^{* *}$ & 37 \\
Trigeminal motor & $958 \pm 48(7)$ & $1523 \pm 37(6)^{* * *}$ & 59 \\
Facial & $3820 \pm 211(8)$ & $5251 \pm 330(6)^{* *}$ & 38 \\
Hypoglossal & $1610 \pm 155(4)$ & $2282 \pm 100(5)^{* *}$ & 42 \\
Ambiguus & $406 \pm 6(3)$ & $657 \pm 27(2)^{* *}$ & 62 \\
\hline
\end{tabular}

$\mathrm{MuSK}^{+/+}$and $\mathrm{MuSK}^{-/-}$values are the average \pm SEM. The values in parentheses indicate the number of animals examined $\left(* * p<0.01,{ }^{*} * *<<0.001\right)$. Percentages (\%) refer to the increase in the number of motoneurons with respect to the controls $\left(\mathrm{MuSK}^{+/+}\right)$.

neural agrin is to trigger AChR clustering, these results strongly suggest that the synaptic disruption caused by the disorganization of AChRs enhances motoneuron survival.

\section{Motoneuron survival in MuSK-deficient mice}

The results obtained in agrin-deficient mice suggested that the increased motoneuron survival was caused by synaptic impairment in the periphery; however, a central effect caused by agrin deficiency could not be dismissed. To investigate this alternative, we analyzed the number of motoneurons in MuSK null mutant mice. MuSK is a transmembrane tyrosine kinase, selectively expressed in skeletal muscle in which it is colocalized with AChRs in the postsynaptic membrane (Valenzuela et al., 1995). It is a component of the agrin receptor complex that plays a key role in AChR clustering. MuSK-deficient mice have a phenotype that is similar to, but more severe than, the null mutants for the agrin protein (DeChiara et al., 1996). These animals show no movement in utero, AChR levels are uniformly low over the entire myotube, and there is a total absence of AChR clustering and postsynaptic specialization. As with agrin-deficient mice, MuSK null mutants show extensive axonal branching on peripheral myotubes (DeChiara et al., 1996; Gautam et al., 1999).

We observed that the number of motoneurons was higher in MuSK-deficient animals than in the wild types. The increase in motoneuron number was $37,59,38$, and $42 \%$ in the trochlear, trigeminal, facial, and hypoglossal motor nuclei, respectively (Table 3). In the ambiguus nucleus, MuSK-deficient animals had 62\% more motoneurons than wild-type mice. Together, these results indicate that impairment of AChR clustering, independent of its neural (agrin ${ }^{\Delta z / \Delta z}$ deficiency) or muscle (MuSK deficiency) origin, is sufficient to decrease motoneuron cell death during development.

\section{Motoneuron survival in rapsyn-deficient mice}

A third critical regulator of AChR clustering is a $43 \mathrm{kDa}$ cytoplasmic protein called rapsyn that acts downstream from MuSK and is colocalized with AChRs from the earliest stages of synaptogenesis (Noakes et al., 1993). In the absence of rapsyn, AChRs do not form clusters beneath motor nerve terminals (Gautam et al., 1995). In several respects, however, the phenotype of rapsyn mutants is less severe than that of agrin or MuSK mutants. First, although axons fail to form terminal arbors, their pattern of branching was less extensive than in agrin- or MuSK-deficient mice (Gautam et al., 1999). Second, some postsynaptic components, including MuSK itself, aggregate in the absence of rapsyn, forming a rapsyn-independent synaptic scaffold (Apel et al., 1997). Third, synapse-associated myonuclei remain transcriptionally specialized in the absence of rapsyn and transcribe AChR 
Table 4. Number of motoneurons in rapsyn null mutant mice (rapsyn $^{-1-}$ ) and their wild-type littermates

\begin{tabular}{lccl} 
& Rapsyn $^{+/+}$ & Rapsyn $^{-/-}$ & $\%$ increase \\
\hline Trochlear & $178 \pm 22(5)$ & $223 \pm 16(7)$ & 25 \\
Trigeminal motor & $1119 \pm 59(7)$ & $1511 \pm 35(8)^{* * *}$ & 35 \\
Facial & $4572 \pm 150(7)$ & $5238 \pm 173(8)^{*}$ & 15 \\
Hypoglossal & $2237 \pm 61(3)$ & $2567 \pm 96(3)^{*}$ & 15 \\
\hline
\end{tabular}

Rapsyn $^{+/+}$and rapsyn ${ }^{-/-}$values are the average \pm SEM. The values in parentheses indicate the number of animals examined $\left({ }^{*} p<0.05\right.$, $\left.{ }^{* * *} p<0.001\right)$. Percentages $(\%)$ refer to the increase in the number of motoneurons with respect to the controls $\left(\right.$ rapsyn $\left.^{+/+}\right)$.

Table 5. Size of motoneurons in $\mu \mathrm{m}^{2}$ in MuSK null mutant mice $\left(\mathrm{MuSK}^{-/-}\right)$and their wild-type littermates

\begin{tabular}{llll} 
& $\operatorname{MuSK}^{+/+}\left(\mu \mathrm{m}^{2}\right)$ & $\mathrm{MuSK}^{-/-}\left(\mu \mathrm{m}^{2}\right)$ & $\%$ increase \\
\hline Trochlear & $151 \pm 4(50)$ & $161 \pm 4(81)$ & +7 \\
Trigeminal motor & $210 \pm 4(105)$ & $218 \pm 4(100)$ & +4 \\
Facial & $205 \pm 5(91)$ & $212 \pm 5(93)$ & +3 \\
Hypoglossal & $197 \pm 5(85)$ & $193 \pm 5(106)$ & -2
\end{tabular}

$\overline{\text { Motoneurons from three wild-type animals and three } \mathrm{MuSK}^{-/-} \text {mice were analyzed }}$ for each of four cranial nuclei. $\mathrm{MuSK}^{+/+}$and $\mathrm{MuSK}^{-/-}$values are the average \pm SEM. The values in parentheses indicate the number of cells examined. Percentages (\%) refer to the increase in the number of motoneurons with respect to the controls $\left(\mathrm{MuSK}^{+/+}\right)$.

subunit and other synaptic genes at higher levels than nonsynaptic nuclei in the same cytoplasm (Gautam et al., 1995, 1999; Apel et al., 1997). Finally, some muscle movement is detectable in rapsyn $^{-1-}$ embryos, and neonates are able to breathe for a few hours (Gautam et al., 1995).

We analyzed cranial motoneurons in newborn rapsyn-deficient mice. As in the other mutant mice previously analyzed, an increase in the number of cranial motoneurons was observed (Table 4). Rapsyn-deficient mice showed a significant increase in the number of facial (15\%), hypoglossal $(15 \%)$, and trigeminal $(35 \%)$ motoneurons. The number of trochlear motoneurons, although not significantly different from the controls, was also higher $(25 \%)$ in the mutant mice.

\section{Motoneuron aspect and size in mice with synapse disruption}

We performed a detailed analysis of motoneuron size in the trochlear, trigeminal motor, facial, and hypoglossal nuclei in mice lacking MuSK. We found no significant difference in motoneuron size between mutants and wild-type controls (Table 5). Quantitation was less extensive in the other mutants, but we did not observe any striking difference in the size of rapsyn ${ }^{-1-}$ or agrin $^{-1-}$ motoneurons as compared with control animals. Furthermore, the shape and arrangement of the cytoplasm, nucleus, and nucleolus did not differ detectably between any of the mutants and heterozygous or wild-type controls (Fig. 2). The normal morphological features and the size of the mutant motoneurons suggest that they are not atrophic.

\section{Motoneuron survival in MuSK-CNTFR $\alpha$ double null mutant mice}

Mice lacking the cytokine receptor CNTFR $\alpha$ showed a $40 \%$ motoneuron deficit at birth, suggesting that CNTF-like cytokines provide trophic support for motoneurons (DeChiara et al., 1995). Because MuSK-deficient mice showed a $37-59 \%$ increase in motoneurons (Table 3), we were interested in knowing whether
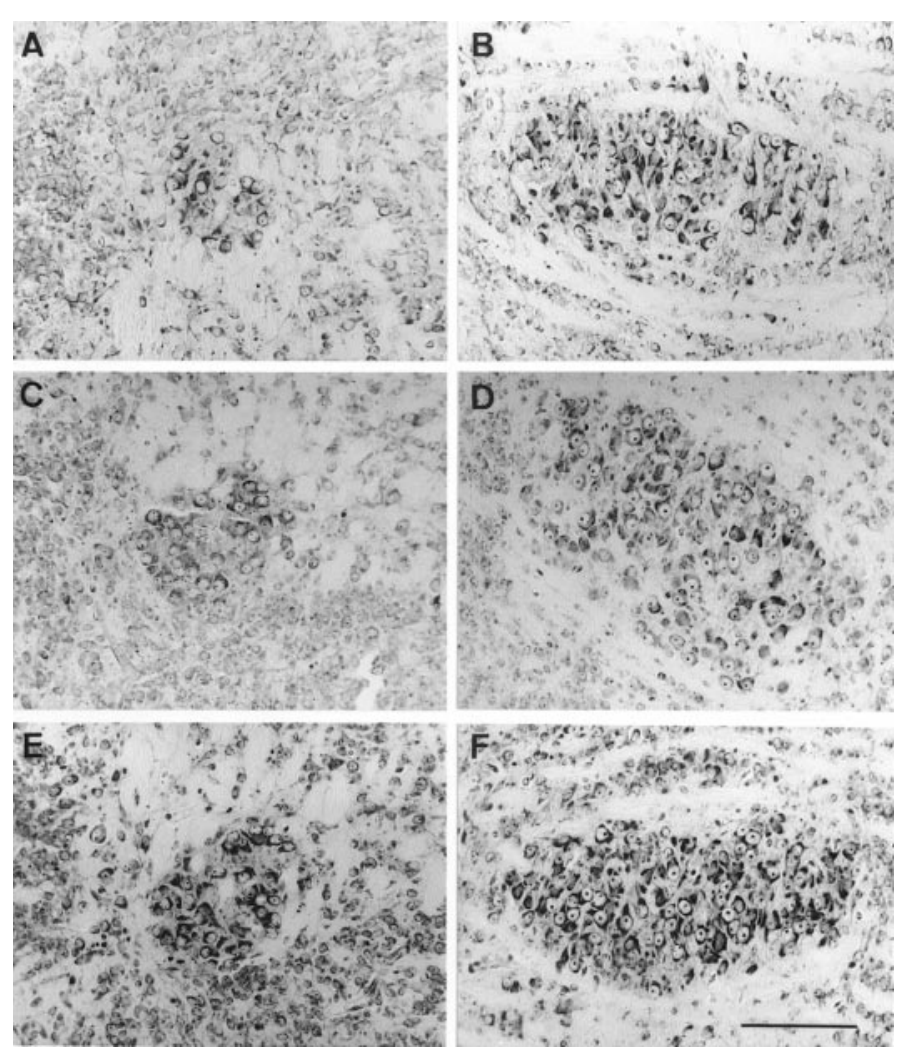

Figure 2. Micrographs of the trochlear nucleus $(A, C, E)$ and trigeminal motor nucleus $(B, D, F)$ of wild-type $(A, B), \operatorname{MuSK}^{-1-}(C, D)$, and rapsyn $^{-1-}(E, F)$ mice. Note the normal morphological features of the motoneurons in the transgenic mice. Scale bar, $100 \mu \mathrm{m}$.

double null mutants that were deficient in both CNTFR- $\alpha$ and MuSK showed a motoneuron phenotype that was characteristic of one of the two mutants. For example, if the motoneurons rescued in the MuSK null mutants were dependent on CNTF-type ligands, then they should die when the CNTF receptor is eliminated. By contrast, if these motoneurons were dependent on neurotrophic factors other than $\mathrm{CNTF}$ receptor ligands, then they should survive despite the absence of the CNTFR $\alpha$.

We crossed heterozygous mice for CNTFR $\alpha$ and MuSK to obtain null mutants with a double deletion. Animals were genotyped using Southern analysis. Null mutant mice deficient in both genes also died at birth, but their morphology appeared to be normal. We counted the number of trochlear, trigeminal, and facial motoneurons in these mice. The double mutants exhibited approximately the same number of motoneurons as the MuSK null mutants alone (Table 6). Therefore, the modifications produced by the absence of MuSK completely overcame those caused by $\mathrm{CNTFR} \alpha$ deficiency.

These results suggest not only that the alterations provoked by synapse disruption are independent of the CNTF receptor pathway but also that these changes are even capable of rescuing motoneurons that would have died normally in the CNTFR $\alpha$ deficient animals. Because of the increased branching of the axons, there may be improved "access" of these excess motoneurons to muscle-derived neurotrophic factors other than CNTF homologs that can now rescue these cells. In addition, the CNTFdependent motoneurons in the $\mathrm{MuSK}^{-/-}$mice may be rescued at a developmental stage when they are still independent of support from a CNTF-like ligand. 
Table 6. Percentage variation in motoneuron numbers in $\mathrm{CNTFR}^{-1-}, \mathrm{MuSK}^{-/-}$, and $\mathrm{MuSK}^{-1-}$ $\mathrm{CNTFR}^{-/-}$with respect to wild-type controls

\begin{tabular}{lllll} 
& Trochlear & Trigeminal motor & Facial & Hypoglossal \\
\hline $\mathrm{CNTFR}^{-1-}$ & n.d. $^{a}$ & $-27 \%^{a}$ & $-41 \%^{a}$ & $-56 \%^{a}$ \\
$\mathrm{MuSK}^{-1-}$ & $+37 \%$ & $+59 \%$ & $+38 \%$ & $+42 \%$ \\
$\mathrm{MuSK}^{-1-} \mathrm{CNTFR}^{-/-}$ & $+40 \%$ & $+48 \%$ & $+24 \%$ & n.d.
\end{tabular}

Percentages $(\%)$ refer to the increase in the number of motoneurons with respect to the controls. n.d., Not determined. ${ }^{a}$ Results described in DeChiara et al. (1995).

\begin{tabular}{|c|c|c|c|c|c|c|c|c|}
\hline & Trochlear & $\%$ & Trigeminal motor & $\%$ & Facial & $\%$ & Hypoglossal & $\%$ \\
\hline WT and $(+/-)$ & $198 \pm 7(29)$ & & $1078 \pm 27(29)$ & & $4315 \pm 111(26)$ & & $1909 \pm 109(10)$ & \\
\hline $\operatorname{Agrin}^{-1-}$ & $271 \pm 27(7)^{* * *}$ & 37 & $1339 \pm 58(6)^{* * *}$ & 24 & $4734 \pm 89(5)$ & 10 & $2412 \pm 98(3)^{*}$ & 26 \\
\hline $\operatorname{Agrin}^{\Delta z / \Delta z}$ & $259 \pm 13(5)^{* *}$ & 30 & $1380 \pm 64(6)^{* * *}$ & 28 & $4964 \pm 169(5)^{*}$ & 15 & $2518(1)$ & 32 \\
\hline Rapsyn $^{-1-}$ & $223 \pm 16(7)$ & 12 & $1511 \pm 35(8)^{* * *}$ & 40 & $5238 \pm 173(8)^{* * *}$ & 21 & $2567 \pm 96(3)^{*}$ & 35 \\
\hline $\mathrm{MuSK}^{-1-}$ & $297 \pm 17(8)^{* * *}$ & 50 & $1523 \pm 37(6)^{* * *}$ & 41 & $5251 \pm 130(6)^{* *}$ & 22 & $2282 \pm 100(5)^{*}$ & 20 \\
\hline Mean of synaptic mutants & $264 \pm 9(27)^{* * *}$ & 33 & $1444 \pm 28(26)^{* * *}$ & 34 & $5079 \pm 110(24)^{* * *}$ & 18 & $2405 \pm 60(12)^{* * *}$ & 26 \\
\hline
\end{tabular}

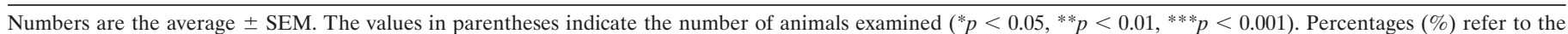
increase in the number of motoneurons with respect to the controls. WT, Wild type.

\section{DISCUSSION}

We addressed the question of whether synapse formation affects the number of motoneurons that survive during development. Several previous studies were consistent with the idea that motoneurons would not survive if they failed to form neuromuscular junctions (Oppenheim et al., 1997). We found, however, that motoneuron number was not decreased in the absence of synapse formation, as might have been expected from previous studies, but actually increased. Although the increased number of motoneurons could result from either an increased motoneuron generation or a decrease in motoneuron death, the former alternative is improbable. MuSK and rapsyn are produced only in the muscle and not in the brainstem or the spinal cord; therefore it seems unlikely that the latter two proteins could affect motoneuron generation. It is therefore highly likely that the increased number of motoneurons results from a decrease in motoneuron cell death.

\section{Cranial motoneurons are differentially affected in "synapse-disrupted" mice}

A common feature of agrin, MuSK, and rapsyn mutant mice is their inability to form neuromuscular junctions (Gautam et al., 1995, 1996, 1999; DeChiara et al., 1996; Burgess et al., 1999). In this report, we show that there is also a decrease in programmed cell death (PCD) in motoneurons in four different cranial nuclei. Although some of the differences are not statistically significant, each of 17 comparisons (Tables 1-4) shows that the mutants have more motoneurons than the controls.

In Tables 1-4, some of the cases of limited differences in the number of motoneurons may have resulted from differences in the genetic background of the mutant mice. To test this possibility, we have combined all of the values of the controls (mice with normal synapses) in the different mutants and used them for a comparison with the experimental values (mice with synapse disruption) (Table 7). Indeed, the number of motoneurons in synapsedisrupted mice differs statistically from mice with normal neuromuscular junctions in the majority of the cases. The $\mathrm{MuSK}^{-1-}$ showed the most elevated percentage of motoneurons followed by the rapsyn null mutants, the agrin ${ }^{\Delta z / \Delta z}$-deficient mice, and finally the $^{\text {agrin }^{-/-}}$animals. With respect to the cranial motor nuclei, the highest percentage of motoneuron survival occurred in the trochlear and trigeminal motor nuclei and the lowest in the facial. The number of hypoglossal motoneurons attained intermediate levels.

The increased survival obtained in the different mutant strains does not rescue all motoneurons from PCD. Considering that at least half of the motoneurons generated during development die during the time of PCD, and because the improved survival varied between 10 and $60 \%$, we can conclude that not all of the motoneurons are affected to the same extent. It is possible that the modifications provoked by synapse disruption are not the same in all of the different muscles and could thus account for the difference in motoneuron survival. The differences could also be explained by a variation in the temporal pattern of PCD in the diverse cranial nuclei, by subtypes of motoneurons that have different trophic factor requirements, or by some residual neuromuscular activity that remains in the mutants.

It is generally considered that there are two different populations of motoneurons, depending on the origin of the muscle cells they innervate: those that innervate somite-derived muscle cells and those that innervate muscle derived from the branchial arches. Our results show that motoneurons innervating both somite-derived (trochlear and hypoglossal) and branchial archderived muscle cells (facial and trigeminal motoneurons) are rescued from cell death when synapses are eliminated. Thus the absence of synapse formation appears to have similar effects on both types of motoneurons.

Although the number of motoneurons affected in the different nuclei of the mutant mice varied, we could not detect any difference in the morphological characteristics of the excess motoneurons as compared with controls. The size of these motoneurons was similar to control animals, suggesting that these cells are in good health and not in the process of undergoing apoptosis.

\section{Theories for increased motoneuron survival during development}

It is now generally accepted that neurotrophic factors play a critical role in the regulation of neuronal survival during devel- 
opment. To explain how neurotrophic factors can be limiting in this context, Oppenheim (1989) proposed a hypothesis based on the access of motoneurons to the neurotrophic factor support by means of increased branching and synaptogenesis on the muscle. This theory also took account of studies showing that chronic muscle blockade in the chick embryo causes increased branching and synaptogenesis, which leads to the rescue of motoneurons from PCD (Dahm and Landmesser, 1988, 1991; Usiak and Landmesser, 1999). Furthermore, in studies using chronic muscle blockade to examine motoneuron survival, there was no dramatic alteration of the synaptic structure (Laing and Prestige, 1978; Pittman and Oppenheim, 1978, 1979), suggesting that the synaptic integrity was not implicated in this phenomenon. In addition, treatments that reduced nerve branching and synapse formation also decreased motoneuron survival (Tang and Landmesser, 1993). Finally, mutant strains of mouse and chicken embryos that exhibit neuromuscular paralysis during development show an increased branching and synapse formation along with an increased number of motoneurons (Oppenheim et al., 1997).

Our results allowed us to eliminate one particular aspect of the access hypothesis; all mutants that we studied have profound deficits in synapse formation, although they differ in the severity of the phenotype. The two different strains of agrin-deficient mutant mice show synaptic disorganization accompanied by AChR clusters that are small in size and often remain outside the central endplate (Gautam et al., 1996, 1999). Rapsyn-deficient mice show an overproduction of AChRs, but there is no cluster formation (Gautam et al., 1995, 1999). The most severely affected mice that we have analyzed, the MuSK null mutants, do not have AChR clustering or any postsynaptic specialization (DeChiara et al., 1996). Despite the absence of synapses, the mice have an increased number of motoneurons. These results suggest that synapses themselves are not the only privileged sites at which trophic communication occurs between muscle and nerve.

If one assumes that increased motoneuron survival occurs because of additional neurotrophic factor support and if synapses are not required for the action of these factors, then the actual location of these uptake sites needs to be considered. The most plausible explanation is that neurotrophic factors act along the entire surface of the axonal branches. Our experiments support the hypothesis that increased branching is responsible for the improved access of the motoneurons to their source of neurotrophic factor sustenance. Although there may be a quantitative relationship between branching and survival, it does not appear to be striking; in the rapsyn mutants, there is less branching than in MuSK or agrin mutants (Gautam et al., 1995, 1996, 1999; DeChiara et al., 1996; Burgess et al., 1999), yet they show similar levels of motoneuron survival. Any relationship between branching and survival would be subtle at best and difficult to prove. An additional complication is that normal innervation patterns vary among muscles, so it would be difficult to devise a measure of excess branching that would be valid for all of the different muscles.

Our results are supported by the observations of Banks et al. (2001) who showed that rapsyn-deficient mice have a significant increase in the number of motoneurons in the spinal cord accompanied by an increase in intramuscular axonal branching. They suggest that one of the mechanisms for regulating motoneuron survival during development may be related to skeletal muscle activity.

\section{Conclusion}

Previous studies have not provided information on whether neuromuscular junction formation plays a role in motoneuron survival. In this report, we demonstrate for the first time that motoneuron survival in mutant mice with disrupted neuromuscular junctions is increased in the absence of synaptogenesis. Our results support the hypothesis (Dahm and Landmesser, 1988; Oppenheim, 1989) that motoneuron survival depends on the access of motoneurons to their target tissue, and we would argue in favor of increased branching of the axons as opposed to synaptogenesis as a means for providing this improved accessibility. In considering what role synapses play in motoneuron survival, it is important to note that we have used mouse mutants in which synapse dysfunction is altered to varying degrees; rapsyn-deficient mice, as opposed to MuSKand agrin-deficient animals, retain a partial organization of their synaptic structure (i.e., synaptic nuclei and clustering of MuSK), and they initially show some muscle movement. Therefore these elementary aspects of synaptic regulation are not involved in motoneuron survival. Finally, studies using double mutants of CNTFR $\alpha$ and MuSK suggest that different subpopulations of motoneurons are involved in either the cell loss (i.e., in the CNTFR $\alpha^{-1-}$ mutants) or the enhanced survival (i.e., in the $\mathrm{MuSK}^{-/-}$animals).

\section{REFERENCES}

Apel ED, Glass DJ, Moscoso LM, Yancopoulos GD, Sanes JR (1997) Rapsyn is required for MuSK signalling and recruits synaptic components to a MuSK-containing scaffold. Neuron 18:623-635.

Banks GB, Chau TNP, Bartlett SE, Noakes PT (2001) Promotion of motoneuron survival and branching in rapsyn-deficient mice. J Comp Neurol 429:156-165.

Burgess RW, Nguyen QT, Son YJ, Lichtman JW, Sanes JR (1999) Alternatively spliced isoforms of nerve- and muscle-derived agrin: their roles at the neuromuscular junction. Neuron 23:33-44.

Dahm LM, Landmesser LT (1988) The regulation of intramuscular nerve branching during normal development and following activity blockade. Dev Biol 130:621-644.

Dahm LM, Landmesser LT (1991) The regulation of synaptogenesis during normal development and following activity blockade. J Neurosci 11:238-255.

DeChiara TM, Vejsada R, Poueymirou WT, Acheson A, Suri C, Conover JC, Friedman B, McClain J, Pan L, Stahl N, Ip NY, Kato AC, Yancopoulos GD (1995) Mice lacking the CNTF receptor, unlike mice lacking CNTF, exhibit profound motor neuron deficits at birth. Cell $83: 313-322$

DeChiara TM, Bowen DC, Valenzuela DM, Simmons MV, Poueymirou WT, Thomas S, Kinetz E, Compton DL, Rojas E, Park JS, Smith C, DiStefano PS, Glass DJ, Burden SJ, Yancopoulos GD (1996) The receptor tyrosine kinase MuSK is required for neuromuscular junction formation in vivo. Cell 85:501-512.

Gautam M, Noakes PG, Mudd J, Nichol M, Chu GC, Sanes JR, Merlie JP (1995) Failure of postsynaptic specialization to develop at neuromuscular junctions of rapsyn-deficient mice. Nature 377:232-236.

Gautam M, Noakes PG, Moscoso L, Rupp F, Scheller RH, Merlie JP, Sanes JR (1996) Defective neuromuscular synaptogenesis in agrindeficient mutant mice. Cell 85:525-535.

Gautam M, DeChiara TM, Glass DJ, Yancopoulos GD, Sanes JR (1999) Distinct phenotypes of mutant mice lacking agrin, MuSK, or rapsyn. Brain Res Dev Brain Res 114:171-178.

Henderson CE (1996) Role of neurotrophic factors in neuronal development. Curr Opin Neurobiol 6:64-70.

Laing N, Prestige M (1978) Prevention of spontaneous motoneuron death in chick embryos. J Physiol (Lond) 282:33P-34P.

Lewin GR, Barde Y-A (1996) Physiology of the neurotrophins. Annu Rev Neurosci 19:289-317.

Noakes PG, Phillips WD, Hanley TA, Sanes JR, Merlie JP (1993) 43K protein and acetylcholine receptors colocalize during the initial stages of neuromuscular synapse formation in vivo. Dev Biol 155:275-280.

Oppenheim RW (1989) The neurotrophic theory and naturally occurring motoneuron death. Trends Neurosci 12:252-255.

Oppenheim RW (1991) Cell death during development of the nervous system. Annu Rev Neurosci 14:453-501.

Oppenheim RW, Prevette D, Houenou LJ, Pincon-Raymond M, Dimi- 
triadou V, Donevan A, O'Donovan M, Wenner P, McKemy DD, Allen PD (1997) Neuromuscular development in the avian paralytic mutant crooked neck dwarf $(\mathrm{cn} / \mathrm{cn})$ : further evidence for the role of neuromuscular activity in motoneuron survival. J Comp Neurol 381:353-372.

Pittman R, Oppenheim RW (1978) Neuromuscular blockade increases motoneuron survival during normal cell death in the chick embryo. Nature 271:364-366.

Pittman R, Oppenheim RW (1979) Cell death of motoneurons in the chick embryo spinal cord. IV. Evidence that a functional neuromuscular interaction is involved in the regulation of naturally occurring cell death and stabilization of synapses. J Comp Neurol 187:425-446.

Sanes JR, Lichtman JW (1999) Development of the vertebrate neuromuscular junction. Annu Rev Neurosci 22:389-442.

Sanes JR, Apel ED, Burgess RW, Emerson RB, Feng G, Gautam M, Glass D, Grady RM, Krejci E, Lichtman JW, Lu JT, Massoulie J, Miner JH, Moscoso LM, Nguyen Q, Nichol M, Noakes PG, Patton BL, Son YJ, Yancopoulos GD, Zhou H (1998) Development of the neuromuscular junction: genetic analysis in mice. J Physiol (Paris) 92:167-172.

Tang J, Landmesser L (1993) Reduction of intramuscular nerve branch- ing and synaptogenesis is correlated with decreased motoneuron survival. J Neurosci 13:3095-3103.

Terrado J, Monnier D, Perrelet D, Sagot Y, Vejsada R, Mattenberger L, King B, Kato AC (2000a) NGF-induced motoneuron cell death depends on the genetic background and motoneuron sub-type. NeuroReport 11:1473-1477.

Terrado J, Monnier D, Perrelet D, Vesin D, Jemelin S, Mattenberger L, King B, Kato AC, Garcia I (2000b) Soluble TNF receptors partially protect injured motoneurons in the postnatal CNS. Eur J Neurosci $12: 1-5$.

Usiak MF, Landmesser LT (1999) Neuromuscular activity blockade induced by muscimol and $d$-tubocurarine differentially affects the survival of embryonic chick motoneurons. J Neurosci 19:7925-7939.

Valenzuela DM, Stitt TN, DiStefano PS, Rojas E, Mattsson K, Compton DL, Nunez L, Park JS, Stark JL, Gies DR, Thomas S, Le Beau MM, Fernald AA, Copeland NG, Jenkins NA, Burden SJ, Glass DJ, Yancopoulos GD (1995) Receptor tyrosine kinase specific for the skeletal muscle lineage: expression in embryonic muscle, at the neuromuscular junction, and after injury. Neuron 15:573-584. 